ON THE PRESENCE OF

\title{
SULPHUR IN CYSTIC OXYDE,
}

\author{
AND \\ AN ACCOUNT OF A \\ CYSTIC OXYDE CALCULUS. \\ (IN THE MUSEUM OF UNIVERSITY COLLEGE, LONDON.) \\ BY HENRY BENCE JONES, B.A., \\ OF TRINITY COLLEGE, CAMBRIDGE. \\ Communicated by CHaRles haWkins, Ese.
}

READ FEBRUARY 25TH, 1840.

ThE discovery of the existence of sulphur in cystic oxyde, by M. Baudrimont, in France, with the confirmation of this observation, by $M$. Thaulow, in Germany, seems to have satisfied chemists abroad of the constancy of that element in this species of calculus. But the well-known accuracy of Dr. Prout, and the apparent impossibility of overlooking the large quantity of sulphur (above 25 per cent.) which these foreign chemists have found, must still cause us at home to hesitate, and at least to suggest, that possibly there may be two similar cystic oxyde calculi, which differ, in the one having more or less of its oxygen replaced by sulphur. Instances are not wanting in which this element is substituted for a part or even the whole of the oxygen 
of an organic principle. Thus Dumas and Pelonze have shown that the volatile oil of mustard consists of $\mathrm{C}_{32} \mathrm{H}_{20} \mathrm{~N}_{4} \mathrm{~S}_{5} \mathrm{O}_{5}$ while in the oil of cloves $\mathrm{O}_{10}$ is found, and no sulphur. (Annales de Chimie, \&c., t. 53.)

An instance of the total substitution of sulphur for oxygen is supplied by mercaptan, which consists of $\mathrm{C}_{4} \mathrm{H}_{6} \mathrm{~S}_{2}$ while alcohol is $\mathrm{C}_{4} \mathrm{H}_{6} \mathrm{O}_{2}$.

Supposing that two compounds so related exist, the names of cystic, and sulphocystic oxydes would, perhaps, best distinguish them.

In opposition, however, to this supposition, we must mention that Pelonze, whose first analysis had agreed with that of Dr. Prout, re-examined the subject after the discovery above-mentioned, and in a communication to Professor Graham, stated that he then found sulphur present.

The analysis by $M$. Thaulow is contained in the twenty-seventh volume of the Annalen der Pharmacie, and is placed in comparison with that of $\mathrm{Dr}$. Prout and Pelonze, as follows :-

Thaulow. Prout \& Preonze. Reckoned. Found. Reckoned. Found.

Nitrogen . . $\begin{array}{llll}11 \cdot 7 & 11 & 11 \cdot 85 & 11 \cdot 67\end{array}$

Carbon . . . $\begin{array}{llll}30.31 & 30.01 & 29.875 & 30 .\end{array}$

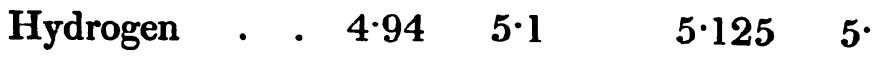

Oxygen . . $\begin{array}{lllll}.26 \cdot 47 & 28 \cdot 38 & 53 \cdot 15 & 53.33\end{array}$

Sulphur . . . 26.58 25.51

whence is deduced the formula $\mathrm{C}_{6} \mathrm{~N} \mathrm{H}_{6} \mathrm{O}_{4} \mathrm{~S}_{2}=$ cystic oxyde : in which the constancy of the sulphur is assumed, though this can only be determined voL. XXIII. 
by the examination of many calculi, and will, for some time, be the object of greatest interest in the analysis of this species.

Such, at least, appeared to me to be the case, when I identified as a cystic oxyde calculus in the collection of calculi belonging to University College, London, the largest specimen probably of this species which exists. It has the form of a large flattened egg, and has been divided. The greatest diameter is two and a quarter inches; the smaller, one and three quarters. The two halves together, after the loss from sawing, grinding, testing, \&c., weigh above 850 grains.

Nothing is known of the history of this specimen of the cystic calculus. The external surface is much broken away ; the highly crystalline structure of this portion usually seen is lost, and what now remains of the outermost layer has a dirty yellowish colour with a waxy lustre, and is, externally, not unlike some biliary calculi.

The surface of the unpolished section is of a yellowish white colour, and in all parts, when closely examined, had a glistening look from minute crystals, which are best seen by candlelight. In the centre there is a small nucleus, around which are two concentric rings, and then for more than a quarter of an inch no more are seen, but in this space the structure appears less compact (having small portions broken away) than that of the more external portion, which last consists of numerous concentric layers, (above forty,) alternately of a whitish yellow and of a darker colour. 
There is no radiation from the centre and no green appearance, which Dr. Marcet thought characterized the larger specimens of this species. When burned, the calculus left a small white ash, and though it in some trials appeared that the central part gave less ash than the more external layers, yet this was not always the case, and in no part was the ash entirely wanting. This ash, on examination, was found to be phosphate of lime.

A portion of the calculus powdered was soluble with effervescence in nitric acid, and gave, when evaporated to dryness, a brownish-black residue, not altered in colour on the addition of ammonia or potash.

Hydrochloric acid dissolved the calculus without efferrescence, and the solution, when quickly evapotated, gave a most disagreeable odour. The crystals formed by slow evaporation of the solution were prismatic.

It was soluble in alkalies and their carbonates, even in the carbonate of ammonia; probably from its containing free ammonia.

The crystals from the solution in ammonia were usually short six-sided prisms or rather tables, but sometimes these were mixed with four-sided ones occurring in clusters.

The mode recommended by Liebig for obtaining pure cystic oxyde was followed in examining the calculus with regard to the presence of sulphur. Of ten grains of the calculus, treated with ammonia, about nine grains were dissolved; which is the proo 2 
portion of cystic oxyde; the remainder being animal matter and phosphate of lime. On the spontaneous evaporation of the ammoniacal solution, crystals were formed, of which five grains were treated with nitric acid free from sulphuric; violent action ensued, after the cessation of which, some crystals of pure nitrate of potash were dropped into the solution, and the whole, after being evaporated to dryness, was gently ignited in a platinum crucible. The white fused mass resulting was alkaline to test paper, and its solution neutralized with nitric acid, gave on the addition of nitrate of baryta an abundant white precipitate, insoluble in the lastnamed acid. This precipitate, collected on a filter, washed, dried, and ignited, weighed 6.94 grains; corresponding to 9576 of sulphur, or above 19 per cent. This is considerably less than the amount given by M. Thaulow, but from the smallness of the quantity operated upon, from its having been examined as crystallized from an ammoniacal solution, and from other sources of error, the result must by no means be considered as throwing any doubt on the correctness of Thaulow's analysis, while it confirms the existence of a large quantity of sulphur in cystic oxyde. Whether the sulphur be variable in quantity, or be sometimes altogether wanting, future observation alone can determine. Meanwhile, it may be remarked that the proximate composition of this substance remains inexplicable. We can find no trace of the sulphocyanogen compounds here ; it bears scarcely any relation to the substances pro- 
duced by the action of sulphurous acid on some of the uric acid compounds ; as, for instance, to thionuric acid $\mathrm{C}_{8} \mathrm{~N}_{3} \mathrm{H}_{7} \mathrm{O}_{14} \mathrm{~S}_{2}$ except that it contains the same elements. Nor does it rank in the amidogen class. It is at present an isolated compound, and offers peculiar interest from its character as a compound base possessing a high atomic weight, from which a long series of compounds may at some future time be derived. It is this peculiarity of composition, and not the mere presence of the sulphur, which renders cystic oxyde so remarkable ; for as $\mathrm{Dr}$. Prout observes, in his Bridgewater Treatise, p. 122, " Not only does sulphur exist in large quantities in the mineral kingdom, but in a greater or less proportion in almost all animal and many vegetable products." Hence the certainty of its introduction into the human system. Accordingly, sulphur in the form of sulphate is found in the blood, bile, and urine, and probably as a sulphuret also in the last. In the hair and brain it exists as a sulphuret, whilst hydrosulphocyanic acid is found in the saliva, and sulphuretted hydrogen in the intestines.

There are a few points respecting the cystic oxyde calculus established by this specimen which deserve notice.

Firstly, The occasional occurrence of concentric rings, and the absence of all radiated structure and green colour. A small cystic oxyde calculus in the possession of Professor Everitt agrees with the above in these points. 
198 MR. JONES ON CYSTIC OXYDE CALCULI.

Secondly, The occasional large size of this species of calculus.

Thirdly, The presence of varying quantities of earthy matter in all parts of the calculus, thus rendering it a compound one.

A beautiful and also very large specimen in St. Bartholomew's Hospital is said to contain small quantities of phosphate of lime and phosphate of ammonia and magnesia. The calculus analysed by M. Thaulow also contained a minute quantity of phosphate of lime.

In conclusion, I have to return my thanks to Professor Graham, in whose laboratory I examined this calculus, and to his assistant, Mr. Fownes, for their kindness, and for the advice which they have given me. 